\title{
Choisir entre plusieurs techniques de fixation des prix : acheter sur catalogue et enchérir sur un même site de commerce électronique
}

Choosing among two different pricing procedures: Online buying and online bidding on the same e-commerce site

\section{Christian Licoppe et Marie-Amélie Picard}

\section{(2) OpenEdition}

Journals

Édition électronique

URL : http://journals.openedition.org/economierurale/3193

DOI : 10.4000/economierurale.3193

ISSN : 2105-2581

\section{Éditeur}

Société Française d'Économie Rurale (SFER)

Édition imprimée

Date de publication : 5 juillet 2005

Pagination : 71-88

ISSN : 0013-0559

Référence électronique

Christian Licoppe et Marie-Amélie Picard, « Choisir entre plusieurs techniques de fixation des prix: acheter sur catalogue et enchérir sur un même site de commerce électronique », Économie rurale [En ligne], 286-287 | Mars-juin 2005, mis en ligne le 05 mars 2007, consulté le 23 avril 2019. URL : http:// journals.openedition.org/economierurale/3193; DOI : 10.4000/economierurale.3193

Ce document a été généré automatiquement le 23 avril 2019.

(c) Tous droits réservés 


\section{Choisir entre plusieurs techniques de fixation des prix : acheter sur catalogue et enchérir sur un même site de commerce électronique}

Choosing among two different pricing procedures: Online buying and online

bidding on the same e-commerce site

Christian Licoppe et Marie-Amélie Picard

\section{Introduction}

1 En dépit des déboires de la "nouvelle économie», le commerce électronique crôit aujourd'hui à un rythme soutenu. Au fur et à mesure que les consommateurs s'habituent à cette médiation marchande, de nouvelles manières de configurer l'échange marchand apparaissent. La consommation sur Internet apparaît comme un processus ramassé dans le temps et l'espace, condensé et focalisé dans la quasi-unité de temps de la session Internet et la quasi-unité de temps de l'écran d'ordinateur connecté. Un consommateur à la recherche d'un bien particulier, par exemple un voyage, peut confronter les offres de plusieurs agences, soit en les consultant à la suite, soit sur le même écran, en ouvrant deux fenêtres de navigation simultanées. Il peut explorer de la même manière différentes mises en marché, en cherchant à acheter un billet d'avion soit sur le site d'une compagnie aérienne, soit chez un discounter pour des voyages pris à la dernière minute, soit encore en se risquant sur des sites d'enchères spécialisés. Dans le monde en dur des magasins, des agences, et des guichets, le même effort d'exploration de l'offre requiert de visiter des lieux différents, ce qui implique souvent des déplacements conséquents, du temps et de la préparation de la part du consommateur. L'effort requis bride l'ampleur de l'exploration, rendant plus rare la visite de lieux de vente trop lointains. 
2 Le commerce électronique a deux conséquences majeures quant à la configuration des rencontres entre l'offre et la demande. D'une part, la croissance rapide du e-commerce met en lumière l'existence d'une multiplicité de modalités pratiques de confrontation de l'offre et de la demande et de détermination des prix, sur l'informatisation desquels il s'appuie (Mirowski et Somefun, 1998), et susceptibles de constituer autant de "configurations algorithmiques", caractéristiques de la microstructure des marchés électroniques (Callon et Muniesa, 2003). Le commerce électronique permet, d'autre part, de rapprocher dans le temps et l'espace ces modalités différentes de mise en marché, de les rassembler sur un même écran, et de les mettre ainsi en concurrence directe dans l'environnement de perception et d'action du consommateur. Différentes formes de commercialisation coexistent alors sur le même site, voire sur la même page. Nous parlerons ici d'effets de voisinage pragmatique et tenterons de rendre compte de la manière dont cette proximité s'inscrit à la fois dans le cadre d'une activité englobante (consommer), d'un cours d'action (explorer l'offre) et d'opérations (les gestes ordinaires de la navigation Web) effectuées de manière routinière dans un espace agencé matériellement à cet effet (le site). Quelles sont les conséquences de ces voisinages pragmatiques nouveaux sur les pratiques commerciales en ligne?

3 Cette question de recherche prolonge dans le contexte du commerce électronique les travaux menés depuis une vingtaine d'années en anthropologie de la consommation et en sociologie économique. Ils se sont attachés surtout à saisir empiriquement la relation entre l'échange marchand et les environnements concrets où il se déroule. Une première tradition a étudié historiquement et ethnographiquement la rencontre entre les consommateurs et les biens dans des lieux et des situations aussi différents que le souk (Geertz, 1979), le marché de province (de la Pradelle, 1996), le supermarché (Miller, 1998), les étalages des marchés aux puces (Sciardet, 2003). L'économie des conventions a introduit dans le champ des sciences économiques et sociales l'analyse des agencements conventionnels sur lesquels s'appuie la qualification des biens (Eymard-Duvernay, 1986; Boltanski et Thévenot, 1991). Une partie de la sociologie économique s'est saisie de ces différents courants de recherche pour éclairer les modalités concrètes de la mise en marché (Barrey et al., 2000 ; Cochoy, 2002), et analyser les dispositifs et les repères au moyen desquels les professionnels du marché ajustent et co-produisent les préférences des consommateurs et les qualités des biens (Hennion et al., 2000; Mallard, 2000; Teil, 2004).

4 Toute mise en marché est aussi mise en espace des biens et des services. Les professionnels du marché sont engagés dans une collaboration et une compétition intense pour produire un arrangement favorable des biens et des services, par exemple au niveau de la disposition de produits similaires dans les linéaires. Dans une perspective écologique de la mise en marché, les effets de proximité intensifient le travail de qualification des produits, parce que le consommateur devient plus sensible à des différences subtiles, pour des biens qu'il peut percevoir et apprécier à l'intérieur d'une même situation. Ecartelé comme l'âne de Buridan entre des produits similaires accessibles d'un seul coup d'œil, il est prêt à basculer au moindre signe (Cochoy, op. cit.). Cette tension est le moteur d'une «économie de l'emballage » qui multiplie les repères susceptibles d'appuyer une qualification du produit, mobilisant un ensemble d'artefacts tels que les comparatifs, packages, guides, descriptifs, modes d'emploi, garanties, chartes de service, normes techniques, labels et certificats. 
5 Au fil de l'histoire des marchés, cette économie des qualités a donné lieu à des situations de confrontation, dans le même espace marchand, à des formes différentes de mise en marché d'un produit: vrac et produits de marque, marques et certificat, etc. Les situations où coexistent des modalités différentes d'appréciation du produit, et particulièrement vente à prix fixes et dispositifs de révélation des prix (marchandages, enchères), ont été plus rares et peu étudiées. Il est néanmoins probable qu'à la fin du XIX ${ }^{e}$ siècle par exemple, au moment où les grands magasins imposaient la vente à prix fixes (Miller, 1981) et où les produits de marque supplantaient la vente en vrac (Strasser, 1989), aient pu coexister une vente en vrac à prix négociable et une vente de produits de marque à prix fixes, dans une proximité spatiale suffisante pour influencer les comportements interactionnels des vendeurs et des acheteurs.

6 Les sites Internet et le commerce électronique confèrent une actualité nouvelle à cette question. Ils multiplient les possibilités de rapprocher dans l'espace d'action du consommateur (la page Internet) non seulement des produits, mais aussi des procédures diverses d'accès, dans lesquelles des produits similaires ne sont pas seulement exposés côte à côte, mais appréciés selon des modalités très différentes. Les protocoles d'enchères sont particulièrement adaptés au monde Internet, comme en témoigne le succès planétaire du site eBay, et la possibilité donnée à un site quelconque de télécharger un module d'enchères préfabriqué et de l'ajuster à sa configuration. Le commerce électronique renouvelle la perspective écologique de la mise en marché en facilitant la construction d'espaces marchands où coexistent vente à prix fixes et dispositifs d'enchères. L'existence d'une telle alternative, qui constitue un consommateur tendu entre deux manières d'évaluer et d'acheter un même bien, est à l'origine du travail fourni par les acteurs pour négocier le cadre de la transaction et la manière dont différentes configurations de demande peuvent y être appréciées.

7 Pour éclairer cette question, nous avons choisi un cas d'étude où ces effets de proximité sont particulièrement aigus. Il s'agit d'une agence de voyages en ligne qui offre le même bien, à partir de la page d'accueil de son site, sous deux formes différentes. En effet, une fois par semaine, cette firme met aux enchères électroniques une série de voyages et de séjours. Or d'un point de vue économique, vente sur catalogue et enchères constituent deux mises en marché distinctes et deux formes différentes de coordination économique, tenues séparées généralement. Le commerce électronique fournit une opportunité, peutêtre encore unique, d'étudier empiriquement de tels effets de "voisinage » et de cocommercialisation entre deux formes de coordination marchande aussi différentes. Les consommateurs se trouvent donc confrontés à la possibilité d'acheter le même bien, sur le même site, souvent dans la même session, sous deux modalités marchandes et à des prix différents. Comme c'est la même firme qui commercialise les biens sous ces deux modalités de la vente catalogue et des enchères, les agents en relation avec les clients interviennent à propos de prestations qui ont pu être achetées d'une manière ou de l'autre, et au service de consommateurs qui ont été exposés simultanément à ces deux possibilités quand ils ont consulté le site.

8 Le voisinage de deux formes de mise en marché sur un même écran constitue un exemple de la manière dont les technologies d'information et de communication saturent de plus en plus l'environnement des acteurs en points d'appui pour agir et interagir (De Fornel, 1994 ; Hutchby, 2000). Les sites Internet manifestent tout particulièrement cette densité en repères ou en prises pour la relance de l'action. Si les pages Web prennent l'apparence d'un document d'écran, dont l'organisation est très largement textuelle, il s'agit 
néanmoins d'un document très particulier, dont la surface " active » ou « interactive » a pour vocation de condenser et de rendre accessible une quantité aussi grande d'informations et de liens possibles que le permet l'exiguïté de l'écran (Jeanneret, 2000). Les portails Internet et les pages d'accueil des sites de commerce électronique sont particulièrement emblématiques de cette tendance à la saturation des environnements en médiations communicationnelles. La difficulté analytique est de rendre compte de manière précise de la relation entre l'agencement spatial des environnements et celle des activités dont ils sont le support, entre la dimension écologique du travail de mise en marché et les pratiques d'échange qui y sont ajustées.

9 Nous allons étudier les conséquences du double voisinage de la vente et des enchères en ligne à trois niveaux :

- Comment les règles des enchères sont recadrées pour être compatibles avec la distribution sur catalogue?

- Comment les comportements de consommateurs et leur rationalité se distribuent-ils selon qu'ils prennent appui sur l'une ou l'autre de ces médiations commerciales, ou sur les deux à la fois (développant alors des stratégies s'appuyant explicitement sur la coexistence des enchères et de la vente catalogue) ?

- Comment la proximité de ces deux formes de vente dans la manière dont les agents traitent les clients crée-t-elle une marge de jeu, particulièrement mise à l'épreuve dans certaines configurations hybrides de la demande? C'est en particulier le cas lorsque certains consommateurs souhaitent partir en nombre plus grand que les billets achetés aux enchères et cherchent à négocier des places supplémentaires.

10 Les questions les plus saillantes se jouent autour de la définition du cadre composite dans lequel une transaction donnée est susceptible de se placer. La proximité des enchères et de la vente sur catalogue engendre une tension entre deux manières de juxtaposer ventes et enchères en ligne : d'un côté, le modèle de la séparation radicale et réglée, et de l'autre une hybridation négociée.

\section{Le voisinage d'écran de deux modalités de mise en marché}

11 Le travail de terrain sur laquelle s'appuie cette étude s'inscrit dans une recherche plus globale sur l'évolution des centres d'appel. Dans le cadre de cet article, nous nous intéresserons à un distributeur du secteur touristique, que nous appellerons ici Travels \& Tours. Possédant un réseau national de points de vente, cette firme est engagée dans une démarche de distribution à grande échelle de prestations de voyage. Pour ses propres produits touristiques, comme pour des vols et des séjours qu'il achète à l'avance par lots à des fournisseurs. Il s'agit de tout vendre, et le plus tôt possible.

12 Egalement doté d'un centre d'appels pour la vente par téléphone, Travels \& Tours développe depuis plusieurs années un site Internet de commerce électronique. Le site est largement dédié à la vente de voyages et de séjours au prix du catalogue. Il propose également un service d'enchères en ligne. Un jour par semaine, le site met aux enchères des vols ou des séjours, en général assez peu de temps avant la date de départ. Les enchères se font par séquence croissante, selon le modèle des enchères anglaises, dans une configuration où seule est visible à un instant donné l'enchère la plus haute ${ }^{1}$. Les managers sont fiers de ce service d'enchères. Ils considèrent avoir été pionniers en 
France, au niveau des voyagistes en ligne, et montrés comme tels dans les grands médias nationaux. Les enchères ont surtout été au début affaire d'image et de communication. À travers les enchères en ligne, la firme a bénéficié d'une visibilité significative dans les médias, en particulier télévisuels, et cherche à se doter d'une image innovante. Le chiffre d'affaires généré par les billets mis aux enchères reste faible par rapport à la vente catalogue, et les enchères ne constituent pas un canal de distribution à part entière.

De ce fait, le système d'enchères a constitué et constitue encore un dispositif de captation des consommateurs, particulièrement utile dans le cadre du commerce sur Internet (Cochoy, 2004). Sans être majeur, son impact économique n'est pas pour autant aujourd'hui complètement négligeable d'un point de vue strictement commercial. Travel \& Tours possède la propriété de n'être pas seulement une firme revendeuse de produits touristiques achetés à des tour opérateurs, mais de jouer elle-même le rôle d'opérateur touristique. Lorsqu'il s'agit de ses propres produits, il est essentiel pour Travel \& Tours de maximiser ses taux de remplissage. Le système d'enchères devient alors un dispositif d'écoulement des stocks. Ces deux usages distincts du système d'enchères, comme dispositif de captation de la clientèle, ou comme dispositif d'écoulement des invendus se combinent pour conférer une relative latitude aux téléconseillers dans le traitement des problèmes relevant des enchères. Le nombre des billets classiques mis aux enchères n'est en général pas assez grand pour qu'on ne puisse prendre le risque de les vendre à bas prix aux enchères (le système est alors traité comme un "pur " dispositif de captation de clientèle). Lorsque le nombre de billets est assez grand pour avoir un impact sur le chiffre d'affaires, c'est en général qu'il faut écouler rapidement les invendus sur un produit maison. Cela fait alors sens de descendre plus bas dans la liste des enchères éligibles, comme nous le verrons plus loin (le système est alors surtout traité comme un dispositif commercial d'écoulement rapide des stocks).

Dans tous les cas, de nombreuses questions soulevées par le comportement des consommateurs enchérisseurs tiennent au fait que les mêmes billets ou séjour peuvent au même moment être réservés et achetés «normalement » sur le site au prix catalogue. A un moment donné deux perspectives de transaction coexistent donc, l'une à un prix fixé, et l'autre à un prix encore à déterminer. De leur côté les téléconseillers du centre d'appels jugent que les billets achetés aux enchères peuvent être vendus tellement bas par rapport au prix catalogue, que c'est plus un cadeau fait à l'enchérisseur heureux ou habile qu'une vente.

15 La page d'accueil du site est majoritairement occupée par l'accès à l'offre catalogue, le moteur de recherche y occupant une place focale. Néanmoins, en haut et à droite, un hyperlien étiqueté "enchères" met en évidence ces deux médiations marchandes. Comme ils sont écrits en mode hypertextuel, il suffit de cliquer dessus pour s'engager dans les modalités de consommation correspondantes. L'écrit d'écran "Enchères » peut être interprété comme une de ces "affordances» dont la théorie écologique de la perception parsème l'environnement (Gibson, 1979), puisque lecture et engagement dans le processus d'enchères semblent s'enchaîner, séparés seulement par le temps d'un clic². Mais un consommateur au corps à corps avec son écran et qui balaye des yeux la page d'accueil est aussi confronté à d'autres prises, telles que le moteur de recherche, ou d'autres liens et images «cliquables" permettant d'acheter un vol ou un séjour de la manière ordinaire ${ }^{3}$. Dans la mesure où une prestation mise aux enchères est également vendue directement au prix habituel, faut-il les envisager comme deux médiations indépendantes, ce qui reviendrait à dire que ce voisinage d'écran ne les affecte en rien? 
Faut-il au contraire les considérer comme un seul et unique dispositif marchand, qui équipe la situation de consommation électronique, et dont les propriétés sont différentes de la simple juxtaposition des médiations marchandes qui le composent? Si ce voisinage d'écran joue un rôle si actif, comment lui donner un sens plus précis? C'est ce que nous allons tenter de faire en en explorant les conséquences, bien au-delà de la surface lumineuse de l'écran.

Cette étude repose sur une série d'entretiens, d'observations et d'enregistrements réalisés dans le centre d'appels qui traite les relations téléphoniques avec les consommateurs en ligne. Ce lieu constitue un point d'entrée privilégié pour reconstituer le travail interactionnel et organisationnel qui constitue la face cachée de ce voisinage d'écran, et qui en est indissociable.

\section{Les règles d'un système d'enchères « contaminé " par la distribution ordinaire}

Les enchères étant uniquement accessibles du site Internet, la responsabilité de leur déroulement incombe au service qui supervise le site et la mise en ligne ("T\&T Online »). Celui-ci sous-traite à un prestataire extérieur la partie logicielle du système d'enchères. Ce dernier enregistre le déroulement, et fournit après clôture une liste ordonnée des gagnants.

18 Vendre aux enchères en ligne des billets et des séjours accessibles également par le catalogue général de Travels \& Tours conduit à un encadrement des prix qui n'aurait pas de raison d'être dans un pur système d'enchères. Vers le bas, les billets sont mis à prix au quart de leur prix catalogue. C'est-à-dire un prix de "dumping", puisque les marges commerciales sur les produits touristiques oscillent typiquement entre 5 et $25 \%$.

On pourrait croire qu'il se déploie là une libre dynamique d'enchères (où l'offreur prend le risque de voir partir son bien à des prix presque symboliques et sans rapport avec sa véritable valeur). En réalité, les gestionnaires du site se voient imposer un prix de réserve (c'est-à-dire un prix minimum en dessous duquel le bien ne peut être vendu), qui n'est pas rendu public, et qu'ils doivent négocier au cas par cas avec les responsables commerciaux. Vers le haut, il arrive que des consommateurs misent plus haut que le prix catalogue $^{4}$, prix auquel celui-ci leur serait accessible s'ils l'achetaient directement sur le site. Mais il n'est pas possible de vendre le vol ou le séjour au-delà du prix catalogue, pour des raisons juridiques. Cette règle n'est pas directement affichée, pour laisser libre jeu à la dimension ludique des enchères compétitives, à l'excitation de voir les prix flamber sans restriction, ce qui contribue à l'efficacité des enchères comme dispositif de captation de la clientèle. Les gagnants en seront avertis, mais après la fin des enchères, lorsqu'ils seront contactés par les agents du centre d'appels. In fine, le prix finalement payé par les enchérisseurs est bridé par le prix catalogue. L'existence d'un prix de réserve, et l'impossibilité de vendre au-delà du prix catalogue bordent la dynamique d'enchères. Cet encadrement des prix traduit la pression qu'exerce sur les enchères la co-existence avec la vente ordinaire.

20 Cette nécessité d'ajustement ne se manifeste pas seulement au niveau de l'encadrement des prix. Ce sont souvent des lots de billets ou de séjours qu'il s'agit d'écouler jusqu'à épuisement de la disponibilité dans une unique enchère où chaque enchérisseur n'a le droit de miser que sur une ou deux places au maximum. Cette configuration se distingue 
des enchères usuelles grand public où chaque bien est singulier (comme dans les enchères d'art), ou encore des cas où le vendeur écoule des lots identiques, mais où chaque lot est séparément mis aux enchères avec des regroupements possibles, comme dans la vente «au cadran» des fraises sur le marché professionnel solognot (Garcia, 1986). De plus, chaque internaute ne peut gagner qu'un ou deux voyages au plus si son enchère est retenue. Dans ce système d'enchères, les vendeurs parcourent la liste des enchérisseurs dans le sens descendant, et leur proposent à tour de rôle les billets au prix de leur enchère, jusqu'à épuisement du stock. Il y a donc le gagnant apparent mais aussi les " éligibles ", c'est-à-dire tous ceux qui seront contactés les uns après les autres par ordre d'enchères décroissant. Les enchères constituent alors un outil commercial pour ajuster de manière réactive et rapide l'offre à la demande. Ce service s'inscrit dans une évolution actuelle de la demande, pour laquelle la proportion de billets et séjours achetés quelques jours avant le départ croît régulièrement depuis plusieurs années, particulièrement chez les internautes (Licoppe et al., 2002). Rapprocher les enchères de la distribution à prix fixes, au sein de la même organisation, prête donc à une forme de rationalisation commerciale dans laquelle les enchères sont de plus en plus utilisées comme manière d'écouler les stocks invendus. Il s'ensuit que le gagnant visible au jeu des enchères n'est pas le gagnant au sens du marché. Celui qui en définitive paiera le moins cher est le dernier des éligibles, dont l'enchère a été tout juste suffisante pour lui permettre d'acheter la dernière place disponible.

21 Ces effets de proximité sont amplifiés par la manière dont l'organisation gère les relations avec les consommateurs. C'est le même centre d'appels qui gère les communications téléphoniques correspondant à toutes les transactions effectuées sur le site (achat, enchères, etc.). Le travail est organisé en équipes polyvalentes (un superviseur, et six à huit téléopérateurs typiquement) : une équipe traite un type d'appel chaque semaine, et change de mission par rotation hebdomadaire. De semaine en semaine, un téléopérateur sera confronté à tous les types de consommateur et de consommation accessibles du site, de sorte que les différents types d'activité et d'interaction leur sont largement présents en mémoire, quel que soit le type de traitement qu'ils sont en train d'effectuer. La proximité spatiale des médiations marchandes sur le site Internet est redoublée par une proximité temporelle du traitement des différents types d'interaction marchande, au niveau de l'organisation séquentielle de l'activité des téléopérateurs.

Dans la gestion des interactions téléphoniques liées aux enchères il convient de distinguer le traitement réservé aux gagnants et celui des éligibles. Les opérateurs contactent directement les gagnants, dont les places ont automatiquement été bloquées à l'avance pour assurer leur disponibilité. Mais l'engagement des gagnants n'est juridiquement effectif que lorsque la firme a reçu un papier (ou un fax) signé de leur part. Toute la difficulté du dialogue consiste pour les opérateurs à obtenir l'expédition de ce formulaire signé, avec des consommateurs qu'ils ne connaissent pas et sur lesquels ne pèsent, à l'inverse des salles d'enchères en présence, que très peu de contraintes de réputation, tout en conduisant le dialogue téléphonique comme s'il était entendu que les gagnants étaient tenus d'honorer leurs enchères. Ils savent néanmoins que s'ils tombent sur un client insatisfait ou très bien informé de ses droits de consommateur, et qui persiste à ne pas honorer son enchère, ils devront faire marche arrière pour ne pas s'exposer à des accusations de vente forcée. Les éligibles ne sont en revanche pas appelés directement. Les opérateurs attendent que ceux-ci (qui ont été notifiés de leur éligibilité 
par courrier électronique) les appellent, pour valider leur enchère en fonction de leur rang et des places encore disponibles.

Toutefois, au fur et à mesure que l'on descend dans les couches successives d'éligibilité, et que le prix d'enchères s'éloigne du prix catalogue, le statut des transactions se fait de manière plus ambiguë. Pour l'enchérisseur, éligible en quatre ou cinquième rang et pour l'opérateur, qui a régularisé avec lui la transaction, s'agit-il d'un jeu, où le client a gagné, et où la firme assume les risques du jeu et accepte de gratifier à ce titre le consommateur? S'agit-il d'une transaction obéissant à une rationalité purement commerciale, une autre façon d'écouler et de solder à la dernière minute des séjours encore invendus ? Il faut à chaque fois renégocier le sens incertain de l'échange ainsi que les rôles et les engagements des acteurs. C'est ce travail interactionnel au-delà des écrans que nous voulons analyser maintenant.

\section{Les comportements du consommateur confronté à la co-disponibilité de deux mises en marché distinctes}

Notre question de départ portait sur le double rapprochement des médiations marchandes, traitées au sein de la même firme et, en outre, accessibles de la même page Internet. La première page du site Internet que nous étudions ici offre une multiplicité de prises, permettant d'amorcer des transactions en ligne. Etre très dense en liens assez fortement redondants entre eux constitue une caractéristique des pages d'accueil des sites marchands et des portails Internet (Beaudouin et al., 2002). Le consommateur en ligne est donc confronté à un environnement complexe, qui autorise des navigations et des cours d'action très différents, pour des efforts comparables. On s'attend par conséquent à ce que la complexité pragmatique des sites conduise à une variété accrue de leurs usages. Le voisinage d'écran de la vente et des enchères complexifie l'écologie de la page d'accueil et se traduit par une dispersion significative des comportements des internautes. C'est ce que montrent les observations que nous avons pu conduire dans le centre d'appels.

$\mathrm{Au}$ fil des appels, les téléopérateurs parcourent toute la gamme des engagements des consommateurs. À un extrême, certains jouent à fond le jeu des enchères, participant activement à l'emballement des offres à la clôture, qui en constitue un des aspects les plus caractéristiques. Ils misent à la dernière minute, et protestent lorsqu'ils découvrent que l'enchère qu'ils avaient cru suffire pour clore l'emballement final avait été surmontée, une minute après l'heure de clôture affichée ${ }^{5}$. À l'autre extrême d'autres traitent les enchères comme une forme de vente et semblent en nier les spécificités en en minimisant les risques. Ils revendiquent dans leurs appels le droit d'être servis, du seul fait qu'ils ont émis une offre supérieure au prix de départ, parce qu'il reste des places disponibles. Ces internautes-là calquent leur comportement sur les attentes et les obligations caractéristiques de la distribution ordinaire. Dans le magasin, quand le produit est là, sous les yeux du consommateur et que celui-ci avance une somme acceptable, il se considère en droit d'être immédiatement encaissé et servi en retour.

L'effet du rapprochement des deux médiations marchandes est de rendre possibles deux manières différentes de combiner les deux pour cadrer l'interaction commerciale, et que révèle la dispersion des pratiques des consommateurs: un cadrage où elles sont radicalement disjointes, et où le consommateur s'engage soit dans le processus d'achat 
soit dans le processus d'enchères (c'est le cas de ces utilisateurs qui misent au-delà du prix catalogue et ignorent cette possibilité de transaction), et un second où ces deux processus sont hybridés, et où les comportements des enchérisseurs sont par conséquent influencés par les pratiques de consommation relatifs à la vente ordinaire à prix fixes (c'est le cas de ces utilisateurs qui enchérissent légèrement en dessous du prix catalogue, cherchant à « acheter » de manière assurée à travers les enchères). Cette coexistence de deux cadrages possibles pour les pratiques de consommation renvoie à la codisponibilité des deux médiations commerciales dans l'espace d'activité des consommateurs.

Certaines tactiques des consommateurs en usent très explicitement. Ces consommateurs réservent ainsi la veille des enchères le billet qui les intéresse et misent le lendemain sur le même billet à un prix bien inférieur. Si leur enchère n'est pas éligible, ils gardent leur billet au prix catalogue. Si elle l'est, ils cherchent à annuler cette réservation. Cette tactique n'est apparente que si les vendeurs sont attentifs, lors de l'annulation du billet à prix catalogue, à l'apparition dans l'historique client de l'autre dossier en cours pour la même destination, correspondant à l'enchère réussie. Certains appels mettent en évidence la grande habileté des utilisateurs à exploiter les ressources qu'offrent les environnements électroniques complexes dans lesquels sont encastrés la vente et les enchères en ligne. Pour enchérir, il faut s'inscrire en donnant un nom et une adresse électronique. Toute la procédure s'articule autour de cette adresse. Des internautes ouvrent alors plusieurs boîtes de messagerie et posent plusieurs enchères différentes, à leur nom, mais avec des adresses électroniques différentes. S'ils se retrouvent éligibles au titre de plusieurs de ces enchères, ils n'honoreront bien sûr que la plus basse.

Cette diversité des tactiques des consommateur est perçue et évaluée par les téléopérateurs. Ils professent ainsi une certaine admiration vis-à-vis des clients « les plus malins ", ceux qui ont choisi de miser sur les produits disponibles en grande quantité, et assez bas pour avoir une forte chance de gagner le vol bien en dessous du prix du catalogue. Ils stigmatisent au contraire pour leur incompétence ceux qui, par ignorance ou excès d'enthousiasme, misent plus haut que le prix catalogue. Il ressort globalement de ces jugements une représentation des consommateurs enchérisseurs comme étant le plus souvent des habitués habiles, et enclins à se montrer « filous ». Dans ce contexte, les conversations téléphoniques sculptent une figure du consommateur, compétent à évoluer dans un environnement complexe et à en exploiter les marges. Les cas les plus extrêmes requièrent la mise en œuvre d'un travail interprétatif particulier Il faut être prompt à pouvoir évaluer le comportement du consommateur, déterminer s'il joue ou non un jeu convenable relativement à l'économie morale de l'échange marchand, et prendre des décisions ajustées, en situation d'interaction. Une charge cognitive importante pèse sur les téléopérateurs dans ces moments de soupçon. Ils doivent interpréter les comportements d'enchères, à la fois rétrospectivement (en utilisant les historiques des enchères qui leur sont accessibles dans les listings informatiques), tout en restant tenus à produire une conversation téléphonique qui convienne.

29 Il y a donc un lien de type écologique entre la complexification des environnements marchands électroniques et la dispersion et la variété croissante des usages des consommateurs, et au développement chez certains de grandes compétences à tenter d'exploiter cette complexité à leur profit. La coexistence de différentes formes de mise en marché dans les environnements de consommation est propice au développement et à la mobilisation de formes adaptées de calcul, tant chez les consommateurs que chez les 
opérateurs. D'une manière plus générale, les rationalités marchandes sont formatées par les artefacts qui outillent les situations d'échange (Callon, 1998).

Une situation, que nous avons vu se reproduire fréquemment dans nos observations, éprouve très directement le cadrage de l'interaction commerciale dans ce contexte de codisponibilité de la vente directe et de la vente aux enchères. Il arrive souvent que des enchérisseurs éligibles se montrent prêts à honorer leur enchère, mais ils souhaitent pouvoir partir en famille ou avec des amis. C'est-à-dire à trois personnes ou plus, alors que le système des enchères ne leur permet d'acheter que deux places à prix d'enchères. Que faire alors? Accepter d'acheter les places supplémentaires au prix du catalogue ? Ceci revient à admettre que les enchères ne constituent qu'un geste commercial limité, encastré dans un univers essentiellement boutiquier. Pourtant, au titre du strict calcul économique, l'entreprise perd à donner ces places supplémentaires aux suivants sur la liste des éligibles, puisque ceux-ci ont forcément misé un peu moins. Ou bien tenter, comme le font presque tous les consommateurs, de convaincre le téléopérateur de leur consentir la faveur de ces billets supplémentaires à prix réduits? Les consommateurs invoqueront alors l'égalité nécessaire de chaque voyageur de leur famille ou de leur groupe face au prix. Ils renonceront souvent à honorer leur enchère initiale s'ils se voient refuser cet accommodement, même s'il n'est pas forcément de leur strict intérêt comptable de le faire.

\section{La demande de billet supplémentaire comme épreuve de la double mise en marché}

\section{Le travail d'évaluation du cadre de transaction comme accomplissement conversationnel}

31 L'échange qui suit est exemplaire d'une telle situation. Il montre également le travail interactionnel à travers lequel se construit le sens de la transaction. Celle-ci constitue véritablement un accomplissement conversationnel. La première partie du dialogue prend une forme assez standardisée. Elle s'étend sur 23 tours de conversation, et se décompose en 3 phases. Une fois passée l'ouverture de conversation, faites de salutations réciproques, la cliente énonce le motif de l'appel: elle a joué une enchère pour deux places sur les Antilles, a reçu un mail lui annonçant qu'elle est éligible et c'est pour cette raison qu'elle rappelle. L'opérateur ratifie sa demande et s'engage dans une séquence assez longue de saisie et de vérification du dossier. Une fois ces vérifications terminées, il redonne la main à la cliente en lui demandant très directement si elle souhaite réserver :

$23 \mathrm{~T}$ (l'opérateur) (bruits de clavier) - Retour le 13. C'est disponible, il y a pas de problème.

(2.0) (deux secondes de silence entre les tours) ${ }^{6}$

$23 \mathrm{~T}$ - Vous souhaitez réserver?

$24 \mathrm{C}$ (la cliente) -Euh... parce que là c'était une enchère pour une ou deux personnes

$25 \mathrm{~T}$ (...) Moi j'ai deux personnes, j'ai Sylvie et Aurore

$26 \mathrm{C}$ - Oui, mais moi nous sommes trois, alors comment ça se passe dans ce cas-là ?

$27 \mathrm{~T}$ - Alors la troisième personne elle sera rajoutée au prix normal

$28 \mathrm{C}-\mathrm{Ah}$ bon!

$29 \mathrm{~T}-$ Oui

$30 \mathrm{C}$ - Comment ça se fait qu'on peut pas jouer plus de deux personnes? 
$31 \mathrm{~T}$ - Parce que le nombre de places est limité, Madame, c'est des ventes de dernière minute, donc il y a un quota de places par participant

$32 \mathrm{C}$ - Ouais mais là ça va pas aller

$33 \mathrm{~T}$ - Bah écoutez je suis désolé, je pourrai pas... je peux pas faire autrement que de facturer deux personnes et en rajouter une troisième avec un prix brochure

$34 \mathrm{C}$ - Au prix brochure? ah ouais !

La cliente saisit cette proposition de concrétisation de sa participation aux enchères comme occasion d'énoncer le problème qui va occuper tout le reste de la conversation : les enchères valent pour une ou deux personnes, mais là, ils sont trois à partir. La question est posée en deux temps, à travers une pré-séquence (Levinson, 1983), initiée par une hésitation qui marque un certain embarras (tour 24), suivie d'une question explicite à son tour de parole suivant. L'usage de ce dispositif conversationnel montre que la cliente s'oriente vers le fait que sa requête puisse être déclinée, rend manifeste qu'elle en est consciente, et projette pour la suite de l'échange le statut d'une négociation.

L'opérateur réagit en énonçant la règle sous la forme d'un principe impersonnel, à savoir que la troisième personne sera rajoutée au prix normal. La cliente remet alors en cause les règles mêmes des enchères (ligne 30) : pourquoi ne peut-on jouer pour le nombre de places dont on a effectivement besoin? Placée après la réaffirmation de la règle par l'opérateur, la question vaut comme une protestation et une contestation de celle-ci. La cliente rappelle ainsi que la transaction prend place dans un contexte centré sur la vente directe de voyages. En tant que consommatrice, la cliente est donc en droit d'attendre que les médiations marchandes accessibles sur le site lui permettent d'acheter le nombre de billets dont elle a besoin, y compris aux enchères. Elle remet en cause la séparation de l'ordre des enchères de celui de la vente ordinaire. L'opérateur, qui l'entend bien ainsi, embraye immédiatement sur une première justification de la règle des quotas (tour 31). Il réaffirme que les enchères ne peuvent être comparées avec la vente ordinaire, parce qu'il s'agit d'une forme de vente de dernière minute où les disponibilités sont limitées. Il suggère qu'il serait moralement tenu d'être équitable vis-à-vis des participants (au titre de l'engagement ludique dans le jeu des enchères), alors même que ceux-ci sont justement inégaux quant au prix qu'ils ont misé (une forme d'inéquité relativement à l'ordre de la consommation ordinaire).

En choisissant de réaffirmer ainsi la règle, le vendeur pré-oriente la conversation vers un refus de tout accommodement, avant même que cette question émerge du déroulement de la conversation. La cliente se ferme, et réaffirme que la situation est inadaptée à sa posture de consommatrice, ce qui, à ce moment constitue une fin de non-recevoir par rapport à la justification précédente du téléopérateur. Celui-ci réagit en affirmant cette fois son incapacité de faire autre chose qu'appliquer la règle, incapacité dont il s'avérera plus tard qu'elle est toute relative. Face à cette intransigeance, la consommatrice réagit par une exclamation sur le prix brochure. Cette référence explicite au prix rend pertinent le développement par le téléopérateur d'une ligne d'argumentation où il compare le prix brochure et le prix d'un billet supplémentaire à prix d'enchères. Sans qu'en soit affirmée la possibilité pratique, cette dernière éventualité ainsi évoquée et thématisée occupe désormais une place légitime dans la conversation :

$35 \mathrm{~T}$ - Le prix normal... bah je peux vous donner le tarif, déjà, si ça reste dans votre budget, alors... (47.0) (bruits de clavier) alors le prix brochure il est à 544 euros 31 (8.0) sachant que vous l'enchère en fait vous l'avez... elle vous était arrêtée à 429 euros plus 56, 
ça nous fait... ça fait en fait 485 euros par personne pour les gagnants, enfin pour l'enchère

$36 \mathrm{C}$ - Bon sinon je, euh

(2.0) (deux secondes de silence)

$37 \mathrm{~T}$ - Là moi je l'ai pas, je vais voir si éventuellement c'était quelque chose qui était proposé sur notre... sur le kiosque, donc c'est des ventes de dernière minute, mais je ne crois pas avoir vu la date de Fort de France, non. Non, je ne peux pas vous le proposer, non

Le prix brochure et le prix enchères sont calculés et énoncés dans le même tour de parole. Dans ce contexte, cette proximité d'énonciation constitue une mise en forme conversationnelle de la proximité des deux prix. Ce tour de parole rend pertinent une reprise à son compte de ces calculs par la cliente, ce qui pourrait constituer l'amorce d'une négociation commerciale. En ne thématisant pas les prix dans son amorce de réponse, au tour 36 , la cliente rend visible le fait qu'elle décline la prise conversationnelle qui lui est offerte. Confronté à cette fermeture, l'opérateur rebondit au tour suivant, en ouvrant une alternative. Il propose d'explorer la possibilité d'acheter moins cher que le prix catalogue une place de dernière minute sur le service "kiosque ». En s'engageant dans la recherche d'une autre forme de résolution, il semble donner, pour la première fois, un signe de solidarité avec la consommatrice. Mais la solution qu'il propose (aller voir du côté du service Kiosque pour les départs en dernière minute) manifeste à nouveau le fait que les enchères ne sont pas un mode de consommation ordinaire, mais une forme de vente à la dernière minute, et pour laquelle l'application stricte de la règle des quotas invoquée plus haut est donc justifiée. La cliente a probablement saisi cette nuance. Lorsqu'il s'avère après recherche qu'il n'y a pas de place disponible à prix de dernière minute pour cette destination et cette date, c'est le moment qu'elle choisit pour embrayer sur une nouvelle forme de remise en cause de la règle et de ses conditions d'application :

$38 \mathrm{C}$ - Parce que c'est toujours deux personnes maximum

(0.5) (cinq secondes de silence)

$39 \mathrm{~T}$ - Bah c'est deux personnes maximum, oui, en fonction du nombre... vous savez le nombre de places est limité donc euh...

$40 \mathrm{C}$ - Même si j'étais arrivée première?

$41 \mathrm{~T}$ - Même si vous étiez arrivée première

$42 \mathrm{C}$ - J'aurais pas eu quoi, j'aurais pas eu trois places?

$43 \mathrm{~T}$ - Ah non, non, on n'alloue pas des places en plus

$44 \mathrm{C}$ - Ah oui, oui

$45 \mathrm{~T}$ - C'est vrai que comme le nombre de places est limité bah on accepte qu'il y ait deux personnes parce que c'est bon... c'est ce qu'il y a de plus général et il faut savoir aussi qu'on peut pas allouer plus de places que ça donc...euh

Après avoir sollicité et obtenu une reformulation de la règle, elle insiste en demandant si la chose aurait été différente au cas où elle ait été la gagnante des enchères («même si j'étais arrivée première? »). Elle montre ainsi sa perception de l'ambiguïté de ce système d'enchères où coexistent gagnants et éligibles, et de la possibilité d'une discrimination des consommateurs en fonction du prix payé. Elle manifeste à la fois ses compétences de consommatrice relativement aux modalités de mise en marché, et sa compétence conversationnelle. Elle sculpte une place possible dans le dialogue pour que l'opérateur explicite la possibilité d'accommodements à la règle. Alors que l'accommodement est non seulement possible mais très présent à l'opérateur (il en conviendra dans l'entretien, 
juste après cet appel), celui-ci choisit de décliner cette possibilité en réaffirmant une fois de plus la règle.

Sa gêne est néanmoins perceptible car il revient encore une fois sur la règle sans avoir été sollicité, pour tenter d'en justifier un des points les plus délicats. Il explique, en effet au tour 45 qu'il tolère que les enchères portent sur deux personnes parce que c'est la configuration de consommation touristique la plus courante. Il reconnaît donc par là que les soi-disant règles des enchères sont influencées par les usages de la consommation, et mine la rhétorique qui consistait à affirmer une règle pour les enchères qui serait autonome par rapport aux modalités de la consommation ordinaire. Au vu de la séquence conversationnelle qui précède, cet aveu rend patent que la décision de l'opérateur est prise. En reconnaissant le caractère ambigu des règles après avoir rendu plusieurs fois manifeste sa volonté de les appliquer, il affirme en creux son refus de tout accommodement, par le tour de parole même qui en concède la possibilité. La cliente ne s'y trompe pas, et s'engage sur la voie de la clôture en affirmant son refus dès lors d'honorer son enchère. Les deux protagonistes collaborent ensuite à une fermeture rapide et polie de la conversation.

Dans les cas comme celui-ci, il reste des places (mais pas trop), le niveau d'enchères est significativement plus bas que le prix normal, mais pas si bas que le séjour apparaisse exagérément bradé. Rien n'est alors acquis d'avance, et comme le montre l'exemple précédent, c'est dans le cours même du dialogue téléphonique qu'enchérisseurs et téléopérateurs posent ce problème des billets supplémentaires à des prix ni trop hauts ni trop bas, et collaborent à faire émerger et se rendre mutuellement visibles, avec plus ou moins de civilité et d'empathie mutuelle, leurs rôles, leurs attentes et leurs obligations réciproques, en débouchant sur une prise de position mutuellement ratifiée sur le prix du billet supplémentaire.

\section{L'épreuve du billet supplémentaire et l'économie morale de l'échange}

Comme nous l'avons vu dans l'exemple précédent, si cette épreuve est suscitée par le consommateur, et négociée dans le cours de la conversation téléphonique, la résolution est asymétrique, la décision d'attribuer les billets supplémentaires revenant in fine au vendeur. Nous avons fréquemment observé des interactions téléphoniques dans lesquelles, confrontés à des clients demandant qu'on leur vende des places supplémentaires à prix d'enchères, les téléopérateurs nient absolument pouvoir prendre une telle décision, pour dire ensuite tout le contraire, lorsqu'ils commentent à l'observateur l'interaction qui vient de se dérouler (« Moi ma marge de manœuvre je fais ce que je veux. Enfin, je fais ce que je veux, la règle est stricte, normalement... enfin après c'est moi en fait qui dois statuer »). Face à une telle demande, le téléopérateur se trouve placé devant une alternative: appliquer la "règle" des enchères, et refuser de vendre les places supplémentaires à un prix autre que le prix catalogue, au risque de voir ces enchérisseurs refuser, et de devoir ensuite vendre les mêmes places aux éligibles qui les suivent dans la liste, à un prix finalement moindre. Ou bien au contraire consentir le passe-droit demandé, et vendre la place supplémentaire au prix de l'enchère, en renonçant à la différence entre le prix catalogue et l'enchère concernée. Dans le premier cas, il maintient bien séparées les coordinations marchandes, ventes et enchères, tandis que dans le second il les laisse s'hybrider et se "contaminer" un peu plus encore. La négociation de billets supplémentaires par les enchérisseurs constitue donc une épreuve 
qui, parce qu'elle requiert de qualifier la forme même de la transaction, combine des arguments moraux et des principes comptables.

Les deux termes de l'alternative sont évalués en fonction de critères tels que l'écart entre le prix de l'enchères et le prix catalogue. $\mathrm{Si}$, du point de vue du vendeur, le prix d'enchère est proche du prix catalogue, et s'il reste bien sûr des places disponibles, il lui est plus facile de traiter la transaction correspondante comme une "quasi-vente" à prix fixe. L'enchérisseur est dans ce cas presque un client (celui qui achète au prix catalogue), dont la demande justifie un effort particulier, d'autant plus grand que le voyage ou le séjour en jeu est cher. Dans les commentaires recueillis après les dialogues téléphoniques, les vendeurs se déclarent prêts à céder aux demandes des enchérisseurs et à assouplir les règles, lorsque leur mise est à peine inférieure au prix habituel : «Il y a des clients ... alors qu'il restait qu'une place ou un truc comme ça, j'ai appelé le siège. C'est des clients enchères hein, j'ai pas à me casser la tête autant! ... Des séjours Kenya tout ça... Parce que j'ai vu en fait qu'au niveau prix séjour ils étaient pas très loin du prix normal». Dans une telle configuration, consentir le billet supplémentaire à prix d'enchères au client qui en fait la demande, ne constitue qu'un écart minime vis-à-vis de la stricte rationalité comptable. Celui-ci peut être négligé, car dans ce cas de figure, l'enchérisseur est, moralement, presque un acheteur, avec des droits et devoirs similaires (dont celui d'acheter le nombre de places qu'il souhaite à un prix donné).

Lorsqu'en revanche l'écart est trop grand, que le prix d'enchères est sans rapport avec le prix habituel, les vendeurs considèrent qu'en honorant l'enchère, l'entreprise faisait déjà un cadeau au consommateur. Demander une place supplémentaire c'est comme revendiquer d'une manière abusive les droits et la posture d'un client ordinaire, et ils tendent alors à refuser l'accommodement, au risque que le client se désiste et de devoir se rabattre sur des enchérisseurs de rang moins élevé. Dans ce cas fréquent (intransigeance $\mathrm{du}$ vendeur et désistement du client), l'entreprise aura pourtant légèrement perdu au plan strictement comptable. L'argument économique n'est donc pas tout, d'une part parce que les vendeurs ne sont pas incités à faire du chiffre sur les enchères, sur le mode de la prescription individuelle en vigueur dans le cas de la vente par catalogue ${ }^{7}$ et, d'autre part, parce qu'un autre répertoire d'évaluation est en jeu, d'ordre moral. Celui-ci indique quand il est légitime ou non pour l'enchérisseur de demander un passe-droit sur les places supplémentaires, en fonction du gain que son succès aux enchères lui procure, par rapport à un achat à prix catalogue. Les enchérisseurs eux-mêmes intériorisent cette économie morale des situations marchandes. Plusieurs fois, des consommateurs ont demandé si c'était parce qu'ils n'étaient pas les gagnants qu'on leur refusait de leur vendre des billets supplémentaires au même prix. En ce sens, consentir à la demande du client, c'est risquer de rendre légitime un cadre de transaction qui constitue un précédent, et transforme durablement, au-delà de la transaction en cours, les attentes des clients vis-à-vis de la firme, et les rôles mutuels des acheteurs et des vendeurs. Plusieurs de ceux-ci avouent hésiter à consentir des passe-droits trop voyants, même si cela faciliterait leurs interactions avec les clients, dans la mesure où les consommateurs risqueraient la fois suivante de réclamer à nouveaux les mêmes avantages, voire même sur un mode litigieux dont le traitement leur incomberait.

C'est dans les situations intermédiaires que l'ambiguïté est la plus grande. Ce sont les cas où il reste des places (mais pas trop), où le niveau d'enchères est significativement plus bas que le prix normal, et pas si bas que le séjour apparaisse bradé, et comme constituant une remise en cause de l'éthos du télévendeur. Rien n'est acquis, et une grande partie du 
travail de qualification de la transaction trouve alors un point d'appui dans le cours de la conversation elle-même, dont le déroulement contingent fournit des indices supplémentaires. Enchérisseurs et téléopérateurs exploitent l'ordonnancement séquentiel de la conversation et les outils informatiques qu'ils peuvent simultanément mobiliser comme des ressources pour négocier avec plus ou moins de civilité et d'empathie mutuelle, leurs rôles et leurs engagements, leurs attentes et leurs obligations réciproques, vis-à-vis de cette demande de billets additionnels.

Enfin, parce que celle-ci constitue une épreuve du cadre de la transaction qui engage non seulement une rationalité comptable mais aussi des positions morales, les cas les plus délicats ne se limitent pas à une confrontation conversationnelle entre un consommateur et un téléopérateur. Ils mobilisent un collectif distribué que les téléopérateurs, en dépit de leur autonomie, et à cause de leur autonomie, sollicitent dans les cas les plus difficiles. Ceux-ci sont commentés à la cantonade ${ }^{8}$, avec leurs voisins et avec les superviseurs, et enfin pour ceux qui font particulièrement problème, discutés directement avec les responsables du site de commerce électronique. Les dialogues entre opérateurs, superviseurs et gestionnaires du site sont l'occasion d'une explicitation partagée du cas, des cours d'action possibles, et de la qualification et l'évaluation de ceux-ci. Cette publicisation des cas et de leur évaluation constitue le centre d'appels en arène, et l'organisation en collectif solidaire pour traiter les cas les plus délicats, et stabiliser les modes de traitement qui conviennent.

\section{Conclusion}

L'interface du site de commerce électronique étudié ici présente sur une même page deux formes de mise en marché très différentes, les enchères et la distribution à prix fixes. S'il est fréquent de pouvoir acheter un même bien mis en marché à des prix différents, c'est habituellement en des lieux différents et auxquels il est impossible d'accéder simultanément. Aller à la fois dans un magasin, faire les soldes ou les enchères requiert beaucoup de temps et d'effort : les coûts qui accompagnent la recherche des informations permettant de décider quelle modalité de consommation est la plus rentable sont en général prohibitifs. La concurrence entre les différentes mises en marché est en ce sens imparfaite. L'interface du site de commerce électronique rétrécit le temps et l'espace en plaçant simultanément les accès à ces deux formes de médiations marchandes dans l'espace de perception et d'action du consommateur. La comparaison ne demande plus qu'un effort négligeable, de sorte que du point de vue d'un consommateur intéressé à un bien, les deux formes de mise en marché sont en situation de concurrence directe. Cette présence mutuelle de la vente sur catalogue et de la vente aux enchères pèse alors sur les formes de l'échange.

Elle s'accompagne tout d'abord d'un déplacement et d'une complexification des règles $\mathrm{du}$ jeu. Le déroulement des enchères est affecté par la proximité avec la vente ordinaire. Les pratiques des consommateurs se diversifient, depuis celui qui se prend trop au jeu des enchères jusqu'à celui qui le nie et ne veut y voir qu'une vente ordinaire. Certains consommateurs manifestent une réelle compétence pour tenter de tirer avantage de la multiplicité des prises qu'offre le système socio-technique dans lequel la transaction est encastrée. Le travail interprétatif des vendeurs est alourdi, puisqu'il leur faut en permanence évaluer à partir des historiques électroniques et des conversations 
téléphoniques les intentions et la posture des consommateurs, dans des configurations plus variées.

Le travail interprétatif et les marges de manœuvre des vendeurs jouent un rôle d'autant plus important qu'une des propriétés caractéristiques du voisinage d'écran est de produire des situations particulièrement incertaines, ambiguës pour les participants, et dont le cours met à l'épreuve la séparation de la vente et des enchères. C'est le cas lorsqu'un couple éligible pour acheter deux places à relativement bas prix aux enchères souhaite acheter deux places supplémentaires pour ses enfants. Selon que le vendeur prend l'initiative de les vendre au prix catalogue (au risque de voir ces consommateurs refuser d'honorer l'enchère) ou au prix d'enchères, la mise en marché combine différemment ventes et enchères: dans le premier cas, ces deux médiations sont juxtaposées, simultanément accessibles mais selon des conventions qui limitent toute interférence, dans le second elles s'hybrident. Cette situation fait épreuve et doit être tranchée de la manière qui convient. De la même manière que la sociologie économique a montré comment le choix entre des produits similaires était souvent appuyé par des repères qualifiant les produits afin de guider la décision des consommateurs et d'en limiter le caractère aléatoire, le choix d'un cadre de transaction dans le cas où deux modes d'appréciation des mêmes produits sont disponibles émerge d'un travail interactionnel et organisationnel qui contribue à qualifier les termes de l'alternative. Les points d'appui pour qualifier le cadre de transaction dans ces situations d'épreuve incertaines se trouvent donc à la fois dans des répertoires d'évaluation comptables et moraux applicables aux différentes réponses possibles, dans des repères contextuels constitués au cours d'une interaction téléphonique équipée par les outils informatiques, et dans un travail d'organisation reposant sur un collectif éclaté, dont la mobilisation, pendant et après l'interaction, constitue une ressource pour faire face et stabiliser des pratiques de traitement approprié des appels téléphoniques.

Dans le cas étudié ici, le voisinage d'écran de la vente et des enchères complexifie les formes de l'échange. Il rend pertinente et possible une vaste gamme de situations d'échange dont la gestion et l'évaluation configure d'un côté un consommateur plutôt habile et volontiers porté sur la combine et de l'autre un vendeur plutôt subtil dont la marge de manœuvre et de décision est accrue. Certaines demandes appuyées sur la codisponibilité de deux manières d'apprécier les produits requièrent des réponses adaptées, qui s'accompagnent d'un développement des compétences et de l'activité des professionnels du marché. La densité des sites de commerce électronique en prises permettant de s'engager dans des formes variées d'achat semble donc faire travailler considérablement et même enrichir l'ordre social dans lequel les pratiques de consommation sont encastrées. Ce point est d'autant plus important que de nombreuses études de sociologie du travail ont souligné les tendances au renforcement de la rationalisation, voire de la taylorisation des pratiques interactionnelles dans les centres d'appels du secteur des services de consommation grand public ${ }^{9}$. Cette étude de cas montre une situation plus nuancée où les modalités de la mise en marché et les formes de travail organisé des professionnels du marché entretiennent une relation de type écologique, que contribue à renouveler les nouvelles technologies et le commerce électronique.

48 La grande distribution a rapproché les biens de consommation similaires en les disposant dans les mêmes linéaires, à portée de main et de regard des consommateurs. Ce voisinage a lancé une dynamique de différenciation qui porte sur l'objet de l'échange, une économie 
des qualités où les produits se distinguent par des caractéristiques continuellement travaillées par les professionnels du marché. Le commerce électronique permet de mettre en présence et en concurrence différentes procédures d'appréciation des produits. Nous avons montré comment l'unité de lieu et d'action ainsi réalisée au moyen des écrans et des technologies électroniques était susceptible de mettre en branle une dynamique de différenciation qui ne porte plus sur les biens, mais sur les manières possibles de les apprécier, et qui pèse sur les formes de coordination et la microstructure des marchés instrumentés par le commerce électronique.

\section{BIBLIOGRAPHIE}

Barrey S., Cochoy F., Dubuisson-Quellier S. Designer, packager et merchandiser : trois professionnels pour une même scène marchande. Sociologie du travail, 2000, 42, p. 457-482.

Beaudouin V., Fleury S., Pasquier M., Habert B, Licoppe C. Décrire la Toile pour mieux comprendre les parcours. Réseaux, 2000, 116, p. 19-51.

Bessy C., Chateauraynaud F. Experts et faussaires. Pour une sociologie de la perception. Metailié, Paris, 1995.

Boltanski L., Thévenot L. De la justification. Les économies de la grandeur. Paris, Gallimard, 1991.

Boutet J. Le travail devient-il intellectuel ? Travailler, 2001, 6, p. 55-70.

Bowlby R. Carried Away. The Invention of Modern Shopping. University of Columbia Press New York, 2001.

Buscatto M. Les centres d'appels, usines modernes? Les rationalisations paradoxales de la relation téléphonique. Sociologie du travail, 2002, 44, p. 99-117.

Callon M. The Embeddedness of Economic Markets in Economics. In M. Callon (Ed.) "The Laws of the Markets", Blackwell, Oxford, 1998, p. 1-57.

Callon M., Muniesa F. Dispositifs collectifs de calcul. Réseaux,2003,122, p. 189-233.

Cochoy F. Une sociologie du packaging, ou l'âne de Buridan face au marché. Presses Universitaires de France, Paris, 2002.

Cochoy F. La captation des publics entre dispositifs et dispositions, ou le petit chaperon rouge revisité. In F. Cochoy (Ed.), La captation des publics, Presses Universitaires du Mirail, Toulouse, 2004, p. 11-68.

Cousin O. Les ambivalences du travail. Les salariés peu qualifiés dans les centres d'appel. Sociologie du travail, 2002,44, p. 499-520.

De Fornel M. Le cadre interactionnel de l'échange visiophonique. Réseaux 1994, 64.

De La Pradelle M. Les Vendredis de Carpentras. Faire son marché à Paris ou ailleurs. Fayard, Paris, 1996.

Dodier N. Leçons politiques de l'épidémie de sida. Editions de l'EHESS, Paris, 2003.

Eymard-Duvernay F. La qualification des produits. In R. Salais et L. Thévenot (Eds.), « Le travail : marchés, règles, conventions ", INSEE-Economica, Paris, 1986. 
Garcia M.-F. La construction sociale d'un marché parfait. Actes de la rechercheen sciences sociales, 1986,65 , p. 2-15.

Geertz C. Suq. The Bazaar Economy in Sefrou. In C. Geertz C. et L. Rosen (Eds), "Meaning and Order in Moroccan Society. Three Essays in Cultural Analysis", Cambridge University Press, Cambridge, 1979.

Gibson J.-J. The Ecological Approach to Visual Perception. Houghton Mifflin, Boston, 1979.

Hennion A., Maisonneuve S., Gomart E. Figures de l'amateur. Formes, objets, pratiques de l'amour de la musique aujourd'hui. La documentation française, Paris, 2000.

Hutchby I. Conversation and Technology. Polity, Cambridge, 2000.

Hutchins E. Cognition in the Wild. M.I.T. Press, Cambridge, Mass., 1995.

Jeanneret Y. Y a-t-il (vraiment) des technologies de l'information? Presses du Septentrion, Lille, 2000.

Levinson S. Pragmatics. Cambridge University Press, Cambridge, 1983.

Lave J. Cognition in Practice. Cambridge University Press, Cambridge, 1988.

Licoppe C., Pharabod A.-S., Assadi H. Contribution à une sociologie des échanges marchands sur internet . Réseaux, 2002, 116, p. 97-140.

Mallard A. La presse de consommation et le marché. Enquête sur le tiers consumériste, Sociologie du Travail, 2000, 42(3), p. 433-456.

Miller D. A Theory of Shopping. Cornell University Press, Ithaca,1998.

Miller M. Le Bon Marché. Bourgeois Culture and the Department Store (1869-1920). Princeton University Press, Princeton, 1981.

Mirowski P., Somefun K. Markets as Evolving Computational Entities. Journal of Evolutionary Economics, 1998, 8, p. 329-356.

Norman D.-A. Les artefacts cognitifs. In « Raisons Pratiques $n^{\circ} 4$ : Les objets dans l'action. De la maison au laboratoire », EHESS, Paris, 1993, p. 15-35.

Sciardet H. Les marchands de l'aube. Economica, Paris, 2003.

Simon H. The Sciences of the Artificial. MIT. Press, Cambridge, 1969.

Strasser S. Satisfaction Guaranteed. The Making of the American Mass Market. Smithsonian Institution Press, Washington, 1989.

Teil G. De la coupe aux lèvres. Pratiques de la perception et mise en marché des vins de qualité. Editions Octarès, Toulouse, 2004.

Thévenot L. Pragmatiques de la connaissance. In Borzeix A., Bouvier B., Pharo P. (Eds.), « Sociologie et connaissances. Nouvelles approches cognitives ", Editions du CNRS, Paris, 1998, p. 101-139.

\section{NOTES}

1. . On caractérise habituellement les enchères selon le sens des prix successifs atteints qui peuvent être ascendants à partir d'un prix de départ très bas (enchères "anglaises »), ou descendants à partir d'un prix de départ très haut (" enchères hollandaises "), et selon leur caractère plus ou moins public. On a ainsi d'un côté les enchères en co-présence, complètement publiques, où chaque participant voit et entend les enchères proposées par tous les autres, et les enchères secrètes, par lettres fermées, que le commissaire-priseur n'ouvre et classe qu'à la date 
de clôture, sans jamais les rendre publiques. On est ici, comme dans beaucoup d'enchères électroniques grand public dans une configuration de publicité intermédiaire, où l'internaute voit le prix atteint à un instant donné et le pseudonyme de l'enchérisseur "gagnant " à ce moment. Sur le site étudié ici, aucune autre information n'est donnée (pas d'accès à l'historique des enchères, ou au nombre d'enchérisseurs en compétition, comme le fait par exemple eBay).

2. Cette description de l'utilisateur aux prises avec son écran s'appuie sur une approche de l'action comme relation entre l'acteur et l'environnement, initiée par les sciences cognitives (Simon, 1969) et développée dans le cadre de la cognition située (Lave, 1988), la cognition distribuée (Norman, 1993 ; Hutchins, 1995) et la sociologie pragmatique (Thévenot, 1998 ; Bessy et Châteaureynaud, 1995).

3. . De plus, dans la liste des produits mis aux enchères, chaque bien est décrit par un lien actif. Si l'on clique sur celui-ci les caractéristiques du bien apparaissent, dont, bien en évidence, le prix catalogue.

4. . Cette éventualité découle de deux comportements de consommation assez différents. Dans l'un, le consommateur propose sa propre valeur pour le bien, le prix qu'il est lui-même prêt à payer, indépendamment du prix catalogue et de la dynamique d'enchères en cours. Dans l'autre, dans les cas où les enchères s'emballent avant la clôture, les consommateurs sont tellement engagés dans le jeu de l'enchère, que le séquencement rapide des enchères dans l'urgence de la clôture les entraîne au-delà du prix catalogue.

5. . La règle particulière de ces enchères, est qu'il est toujours possible de surenchérir une minute après la dernière enchère, même après la date de clôture. Si par exemple, pour une enchère censée se terminer à $17: 00: 00$, j'ai enchéri à $16: 59: 51$, mon enchère peut encore être couverte par une autre à $17: 00: 45$, et celle-ci par une dernière à $17: 01: 38$, de sorte que l'heure effective de passation de l'enchère de clôture sera supérieure à l'heure « officielle » ou affichée (17:00:00 dans cet exemple).

6. . Notation conventionnelle en analyse de conversation.

7. Les vendeurs ne sont pas intéressés aux transactions réalisées à partir des enchères de la même manière qu'aux ventes ordinaires qu'ils parviennent à conclure. Les enchères sont considérées comme une opération de prestige et de communication, et comme un processus auxiliaire de la distribution à prix fixes, plutôt que comme un canal de distribution à part entière. Les téléopérateurs ne touchent aucune commission sur les ventes aux enchères, sauf s'ils parviennent à revendre aux enchérisseurs des prestations supplémentaires aux tarifs ordinaires. En assurant un traitement convenable des enchères, ils manifestent surtout leur engagement personnel dans l'effort collectif pour faire du chiffre, au nom de l'intérêt commercial de la firme. Cette implication indirecte s'accompagne d'un contrôle moindre sur le chiffre d'affaires réalisé par les managers et les téléopérateurs eux-mêmes, dans la mesure où il n'y a pas de commission individuelle en jeu.

8. . Les conversations téléphoniques se traitent dans une configuration de type "open space ", où les opérateurs voisins peuvent entendre ce qui se passe.

9. . Voir par exemple Boutet, (2001); Buscatto (2002); Cousin (2002).

\section{RÉSUMÉS}

Le commerce électronique permet de mettre en présence et en concurrence différentes procédures d'appréciation des produits. Cet article analyse les conséquences auxquelles conduit 
le voisinage sur un même site de tourisme en ligne la vente sur catalogue et de la vente aux enchères. L'unité de lieu et d'action ainsi réalisée au moyen des écrans et des technologies électroniques pèse sur les formes de coordination marchande instrumentée par le commerce électronique. Le déroulement des enchères est affecté par la proximité avec la vente ordinaire. Les pratiques des consommateurs se diversifient. Le travail interprétatif des vendeurs est alourdi, puisqu'il leur faut en permanence évaluer, à partir des historiques électroniques, et des conversations téléphoniques les intentions des consommateurs, dans des configurations plus variées. La co-disponibilité des deux modalités d'achat à partir d'un même écran produit des situations particulièrement incertaines, dont le cours met à l'épreuve la séparation de la vente et des enchères, comme lorsqu'un consommateur éligible pour acheter deux places à relativement bas prix aux enchères souhaite acheter des places supplémentaires pour partir en famille ou entre amis. La dynamique de différenciation ne porte plus sur les biens, comme dans l'économie des qualités, mais sur les manières concurrentes de les apprécier.

E-commerce technologies enable configurations in which different pricing procedures compete for the attention of consumers. This article reports on the case of a travel site which sells the same products either directly at catalogue price or through auctions, with both possibilities being simultaneously accessible from the homepage of the site. It discusses the ways in which the auction process can be said to be « contaminated » by its vicinity to ordinary consumption, and the interactional and organizational work that is required to manage such interferences. Consumer behaviours display increasingly varied patterns that take into account the coavailability of two pricing and buying procedures. The interpretive burden of call center teleoperators is increased, for they must assess such diverse consumption patterns and the motives that lie behind them, while relying heavily on resources such as phone conversations and the electronic traces left by various keyboard-mediated procedures. Some situations that occur repeatedly prove particularly ambiguous, and blur the separation between standard electronic retail and online buying, while leaving a significant margin of autonomy to the teleoperators. Such is the case when a successful online bidder wishes to buy more than the two seats he has a right to buy that way, and argue for the teleoperator to allow him to buy these additional seats also at bid price. When different pricing procedures are simultaneously available in the activity field of the consumer, the dynamics of differentiation does not operate with respect to goods, as is the case in the economy of quality, but on the variety of available pricing and buying procedures.

\section{INDEX}

Code JEL D40 - General, D44 - Auctions, L81 - Retail and Wholesale Trade; Warehousing; eCommerce

Mots-clés : appréciation, commerce électronique, écran, enchères, mise en marché, sociologie économique

Keywords : auctions, E-commerce, economic sociology, Internet, market, pricing, screen

\section{AUTEURS}

\section{CHRISTIAN LICOPPE}

École nationale des télécommunications, Département Economie, Gestion et Sciences humaines 


\section{MARIE-AMÉLIE PICARD}

Université d'Evry Val d'Essonne, UFR de Sciences sociales et gestion 